\title{
Computertomograf spürt Poren im Aluminiumguss auf
}

Ein neues Konzept von Wissenschaftlern des Fraunhofer-Instituts für Betriebsfestigkeit und Systemzuverlässigkeit (LBF) ermöglicht die Entwicklung von zuverlässigen, optimierten Aluminiumgussteilen, deren Qualität erstmals zerstörungsfrei geprüft werden kann. Mithilfe eines Computertomografen (CT) können die Forscher grafisch dargestellte Schwindungsporen in Aluminiumlegierungen abbilden. Auf diese Weise sind sie in der Lage, Fehler in Bauteilen aus Aluminiumguss aufzuspüren.

Die Wissenschaftler entwickelten unter Berücksichtigung von geometrischen Kenngrößen des Defekts ein Parametermodell zur Lebensdauerberechnung. Damit soll es möglich sein, die Schwingfestigkeit von Aluminiumgussteilen auf Basis von zerstörungsfreien Prüfungen quantitativ zu ermitteln. Das Modell lasse sich auf alle Aluminiumgussbauteile anwenden. Die Forscher entwickelten das Modell im AiFForschungsprojekt "Epos - Entwicklung und Integration von Beurteilungskriterien zur Qualitätssicherung und Bauteilberechnung unter Berücksichtigung des Einflusses von Poren und nichtmetallischen Verunreinigungen auf die Schwingfestigkeit von Aluminium-Gusslegierungen" des Bundesverbands der Deutschen GießereiIndustrie (BDG).

Die Forscher ermittelten dreidimensionale Geometrieinformationen der Porositäten, aus denen sie relevante Kenngrößen ableiteten. Anschließend wurde die Wirkung der Porositäten auf die örtlichen Beanspruchungen im Gefüge hinsichtlich Form, Größe und Lage der Poren untersucht wie auch quantifiziert. Im ersten Schritt erstellten die Wissenschaftler Finite-Elemente-Modelle für kugelförmige Poren, die die untersuchten Gefüge aus Sicht der inneren Beanspruchung ingenieurmäßig sinnvoll abbilden. Im Weiteren folgten FEM-Modelle der realitätsnahen Gefügeinhomogenitäten.
Anhand der CT-rekonstruierten Mikrostrukturen der Proben wurden die mikromechanischen Beanspruchungen abgebildet und parametriert, berichten die Darmstädter weiter. Darüber hinaus brachten die Forscher die innere Kerbwirkung in Zusammenhang zu den charakteristischen Geometriekennwerten der Porositäten und leiteten aus diesen Ergebnissen ein parametriertes Lebensdauermodell ab. Um das Modell zu validieren, führten sie an den ungekerbten Proben Schwingfestigkeitsuntersuchungen durch.

Das erarbeitete Lebensdauermodell soll die Ableitung von dreidimensionalen Grenzmusterbauteilen ermöglichen, die die zulässigen Porositäten darstellen. Damit bestehe grundsätzlich die Möglichkeit, die Schwingfestigkeitseigenschaften von Aluminiumgussteilen auf Basis von zerstörungsfreien Prüfverfahren quantitativ ohne Versuche oder FEM-Berechnungen zu bewerten.

\section{BAYER MATERIAL SCIENCE \\ LEICHTBAU-PUR-SYSTEME MIT GLASS BUBBLES}

Glass Bubbles, kleinste hohle Glaskügelchen von $3 \mathrm{M}$, ersetzen in Kombination mit anderen Additiven im neuen Polyurethan(PUR)-System Bayflex Lightweight von Bayer Material Science die bisher als Füllstoff verwendeten Mineralfasern. Auf diese Weise soll eine Gewichtsersparnis von bis zu $30 \%$ erzielt werden können, be-

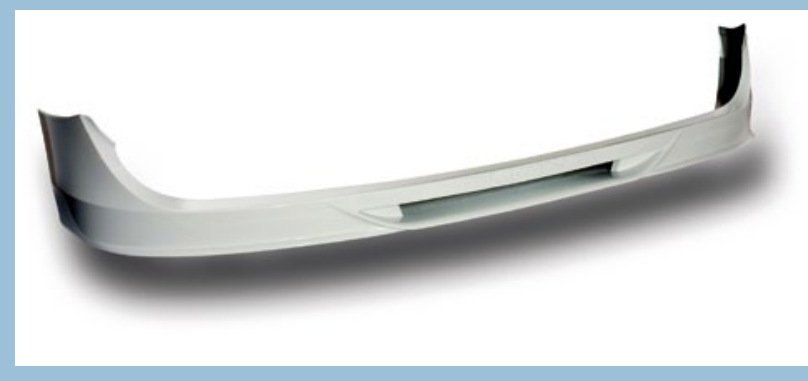

richtet 3M. Und dies bei nahezu identischen mechanischen Eigenschaften. Mit einer Dichte von rund 0,9 kg/l sei das kompakte Material sogar leichter als Wasser.

Aufgrund dessen soll sich das PUR-System vor allem für automobile Leichtbaukonzepte eignen. Der neue Werkstoff ermögliche eine deutliche Senkung von Kraftstoffverbrauch und $\mathrm{CO}_{2}^{-}$ Emissionen sowie bei bestimmten Fahrzeugmodellen zudem eine Verbesserung der Fahrdynamik. Im Elektroauto könne dadurch das zur- zeit noch hohe Gewicht der Batterie besser kompensiert beziehungsweise eine größere Reichweite des Fahrzeugs erreicht werden. Darüber hinaus soll das kompakte Material viel Designfreiheit bei der Fahrzeugkonstruktion bieten. Solche PUR-Bauteile sind laut 3M für die Herstellung von Bauteilen mit Class-AOberfläche geeignet. Die Verarbeitung erfolge mithilfe der Reinforced Reaction Injection Molding-Technik.

Auch der Rennsport sei ein wichtiger Markt für den Werkstoff. In diesem Bereich würden daraus schon heute Stoßfänger, Kotflügel oder Türschweller gefertigt. Das Leichtbaukonzept lasse sich aber auch auf weitere Anwendungsgebiete, zum Beispiel im Nutzfahrzeugbereich, übertragen. 